\title{
Interface Detection in Diffusion Tensor MRI
}

\author{
Lauren O'Donnell ${ }^{1,2}$, W. Eric L. Grimson ${ }^{1}$, and Carl-Fredrik Westin ${ }^{1,3}$ \\ 1 MIT CSAIL \\ 2 Harvard-MIT Division of Health Sciences and Technology, \\ Cambridge MA, USA \\ odonnell@ai.mit.edu \\ 3 Laboratory of Mathematics in Imaging, \\ Brigham and Women's Hospital, Harvard Medical School, Boston MA, USA \\ westin@bwh . harvard.edu
}

\begin{abstract}
We present a new method for detecting the interface, or edge, structure present in diffusion MRI. Interface detection is an important first step for applications including segmentation and registration. Additionally, due to the higher dimensionality of tensor data, humans are visually unable to detect edges as easily as in scalar data, so edge detection has potential applications in diffusion tensor visualization. Our method employs the computer vision techniques of local structure filtering and normalized convolution. We detect the edges in the tensor field by calculating a generalized local structure tensor, based on the sum of the outer products of the gradients of the tensor components. The local structure tensor provides a rotationally invariant description of edge orientation, and its shape after local averaging describes the type of edge. We demonstrate the ability to detect not only edges caused by differences in tensor magnitude, but also edges between regions of different tensor shape. We demonstrate the method's performance on synthetic data, on major fiber tract boundaries, and in one gray matter region.
\end{abstract}

\section{Introduction}

The problem of interface detection in diffusion tensor imaging (DTI) is more complicated than the problem of interface detection in scalar images. This is because there are two types of interface. In DTI data, one would like to detect both interfaces due to changes in tensor orientation and interfaces due to changes in tensor magnitude. Furthermore, it would be ideal to control the relative effects of tensor magnitude and tensor orientation information on the output interfaces.

Potential applications of interface detection in MRI diffusion data are both the same as those in scalar data, and different. Segmentation and registration are two applications that are already known from scalar data. An application that is different is the detection of interfaces that may not be apparent to the human eye. In scalar data, a radiologist is the gold standard for interface detection. However with tensor data, the higher dimensionality of the data and the limitations of any tensor visualization technique may confound human edge detectors. Consequently it is truly useful to study methods of edge detection in 
tensor fields, not just to automate tasks that could be laboriously performed by a human, but to actually enable localization of the interfaces at all.

There are many anatomical interfaces of interest in DTI. The most obvious are tract boundaries, for example the medial and lateral borders of the optic radiation. Another interface that is obvious is the border between the nearisotropic diffusion tensors in cerebrospinal fluid and neighboring tensors, for example those in white matter which have a more anisotropic shape. A very interesting and less obvious type of interface is that within gray matter. For example, it would be clinically interesting if borders of thalamic nuclei were directly detectable.

Related work on tensor interfaces includes one study which presents a method very similar to ours, but without addressing the difference between magnitude and orientation interfaces 44. Their application is level set motion in a tensor field, and their results while nice show mainly interfaces due to tensor magnitude and do not address any anatomical features of interest. An earlier investigation of tensor field edge detection defines the tensor field gradient and its generalized correlation matrix, then applies these to point detection in DTI 14. Another approach to defining edges using local structure in DTI was presented in the context of adaptive filtering [11. Other interface detection in DTI includes the implied interfaces at the borders of tractographic paths [1216]. Finally, any type of segmentation will output interfaces. Work on DTI segmentation includes two methods that have been presented for automatic segmentation of nuclei in thalamic gray matter. The first technique groups voxels using a combined tensor similarity and distance measure [20]. The second method classifies voxels based on their connection probabilities to segmented cortical regions [2]. Both methods produce beautiful results but to our knowledge there has been no work which looks at the local interface structure within the thalamus.

In this paper we present an extension of the method of local structure estimation to tensor-valued images using normalized convolution for estimating the gradients. We produce a new local structure tensor field which describes the interfaces present in the original data. We use the method of normalized convolution to reduce the overall dependence of our results on the magnitude of the tensors, in order to enable detection of both magnitude and orientation interfaces. We present results of our method on synthetic tensor data and anatomical diffusion tensor data.

\section{Materials and Methods}

\subsection{Data Acquisition}

DT-MRI scans of normal subjects were acquired using Line Scan Diffusion Imaging [6] on a 1.5 Tesla GE Echospeed system. The following scan parameters were used: rectangular $22 \mathrm{~cm}$ FOV (256x128 image matrix, $0.86 \mathrm{~mm}$ by $1.72 \mathrm{~mm}$ inplane pixel size); slice thickness $=3 \mathrm{~mm}$; inter-slice distance $=1 \mathrm{~mm}$; receiver bandwidth $=+/-6 \mathrm{kHz} ; \mathrm{TE}=70 \mathrm{~ms} ; \mathrm{TR}=80 \mathrm{~ms}$ (effective $\mathrm{TR}=2500 \mathrm{~ms})$; scan time $=60$ seconds/section. Between 20 and 40 axial slices were acquired 
covering the entire brain. This protocol provides diffusion data in 6 gradient directions as well as the corresponding T2-weighted image. All gradients and T2-weighted images are acquired simultaneously, and thus do not need any rigid registration prior to the tensor reconstruction process. Tensors are reconstructed as described in [17.

In addition, a synthetic dataset was created in matlab for the purpose of demonstrating edge detection based on orientation differences. The dataset consisted of a circle of nonzero diffusion tensors in a "sea" of background 0-valued tensors. The left half of the circle contained tensors whose major eigenvectors pointed vertically, while tensors in the right half of the circle had horizontal principal directions. The purpose of this test data is to demonstrate the results of the method on magnitude and orientation differences in the tensor field.

\subsection{Local Image Structure}

In two dimensions, local structure estimation has been used to detect and describe edges and corners [5]. The local structure in 2D is described in terms of dominant local orientation and isotropy, where isotropy means lack of dominant orientation. In three dimensions, local structure has been used to describe landmarks, rotational symmetries, and motion [73812,13]. In addition to isotropy, it describes geometrical properties which have been used to guide the enhancement and segmentation of blood vessels in volumetric angiography datasets [10] 15], bone in CT images [18], and to the analysis of white matter in DTI [17].

Let the operator $\sum_{a}$ denote averaging in the local neighborhood $a$ about the current spatial location. Then the local structure tensor for a scalar neigborhood can be estimated by

$$
\sum_{a} \nabla I \nabla I^{T}
$$

For multi-valued (vector) data, this formula extends straightforwardly to

$$
\sum_{k} \sum_{a} \nabla I_{k} \nabla I_{k}^{T}=\sum_{a} \sum_{k} \nabla I_{k} \nabla I_{k}^{T}
$$

where $k$ indicates the component. For a tensor field with components $D_{k l}$ the generalized local structure is then estimated by

$$
T=\sum_{a} \sum_{k l} \nabla D_{k l}\left(\nabla D_{k l}\right)^{T}
$$

\subsection{Normalized Convolution}

Normalized convolution (NC) was introduced as a general method for filtering missing and uncertain data [9]19]. In NC, a signal certainty, $c$, is defined for the signal. Missing data is handled by setting the signal certainty to zero. This method can be viewed as locally solving a weighted least squares (WLS) problem, where the weights are defined by signal certainties and a spatially localizing mask. Here we estimate image gradients using normalized convolution. 
A local description of a signal, $f$, can be defined using a weighted sum of basis functions $b_{k}$. Let $B$ denote a matrix where these basis functions are stacked as column vectors. In NC the basis functions are spatially localized by a positive scalar mask denoted the "applicability function," or $a$. Minimizing

$$
\left.\| W_{a} W_{c}(B \theta-f)\right) \|
$$

results in the following WLS local neighborhood model:

$$
f_{0}=B\left(B^{*} W_{a} W_{c} B\right)^{-1} B^{*} W_{a} W_{c} f,
$$

where $W_{a}$ and $W_{c}$ are diagonal matrices containing $a$ and $c$ respectively, and $B^{*}$ is the conjugate transpose of $B$.

The coordinates $\theta$ describing the local signal $f_{0}=B \theta$ are

$$
\theta=\left(B^{*} W_{a} W_{c} B\right)^{-1} B^{*} W_{a} W_{c} f
$$

The estimated coordinates are used in this paper to describe the gradient from planar basis functions, $b_{1}=1, b_{2}=x, b_{2}=y$, and $b_{2}=z$, where $x$, $y, z$ are local spatial coordinates. Since normalized convolution calculates the coordinates of data described locally using this basis, the last three coordinates correspond to the derivative in $x, y$, and $z$ respectively.

\subsection{Subvoxel Gradient Estimation}

We can effectively calculate the gradient on a higher-resolution grid than the voxel resolution, using the separation of data and certainty to our advantage. The goal of using a higher-resolution grid is to increase the ability to detect edges that may be close together on the original grid. To "expand" the initial grid, we insert zero-valued tensors between data points. The operation of the rest of the algorithm is unchanged, since those points are simply treated as uncertain in the gradient computation. Empirically this gives improved results over a two-step process of first interpolating the tensor data and second calculating the local structure.

\subsection{Procedure}

First, for each DTI dataset local structure estimation was performed as described above using gradients from normalized convolution. We employed the trace of the diffusion tensors as the certainty measure. This method emphasizes tensor shape edges over diffusion-magnitude edges and aims to suppress border effects. Then regions of interest were expanded as described above for detection of spatially close edges, and the local structure estimation was run on these regions.

The choice of applicability function depends on the width of the edges of interest. Here we use a Gaussian function, and experiments were performed with standard deviations between one and two mm, and neighborhood sizes (in voxels) 


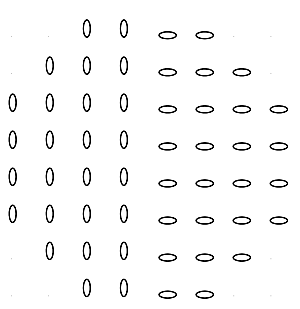

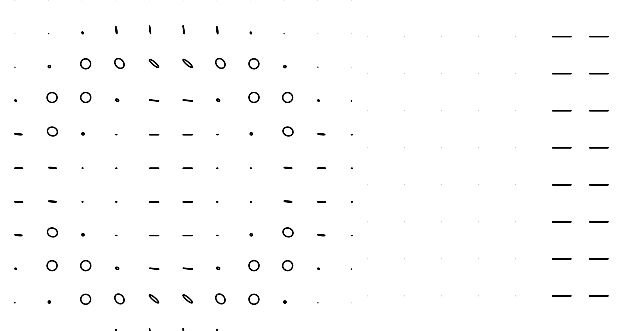

Fig. 1. Simulated data to show unwanted bias in local interface estimation in tensor data close to data borders. The leftmost image shows the input tensor data. The middle image is the local structure tensor estimated without normalized convolution, i.e. with no knowledge of data certainty. Note the unwanted responses on the border of the data, and how this affects the estimation of the interface between the to regions close to the border. The rightmost image shows the shape of the local structure tensors estimated with normalized convolution. Note that the interface is now correctly estimated between regions of tensor data and no border effects are present.

from 9 by 9 by 9 to 21 by 21 by 11 . We aimed to match the variance to the size of the features of interest and to the data resolution. The neighborhood sizes were chosen to allow the Gaussian to fall smoothly to near zero at the boundaries. In order to perform subvoxel gradient estimation we found that inserting one or two zero voxels between known data points was useful. In practice, creating a larger grid than that gave little improvement and was computationally expensive.

\section{Results}

Here we demonstrate the performance of the method on synthetic data and we show selected results from diffusion tensor MRI data.

First we present an experiment showing the performance of the method on the synthetic data described in Section 2.1. The goal is to show that the algorithm will react only to edges that are accompanied by local confidence in the data.

Figure1demonstrates the result: the input tensors have both magnitude-type and orientation-type edges, but only the orientation-type edges are detected by the local structure estimation with normalized convolution. The certainty outside of the "circle" is proportional to the tensor trace which is zero there, so the method does not recognize the border of the circle. It detects only the edge caused by differences in tensor shape.

Figure 2 shows slices through the trace of the local structure tensor at many levels in an axial DTI dataset. Anatomical borders, including the following list, are detected. Note the bilateral cingulate bundles running in an anteriorposterior direction in the top middle slice. In the top right and lower left slice the corpus callosum can be seen. The anterior and posterior limbs of the internal capsule are seen in the lower left slice. Additionally the optic radiation and some brainstem structure can be seen in the lower right slice. 

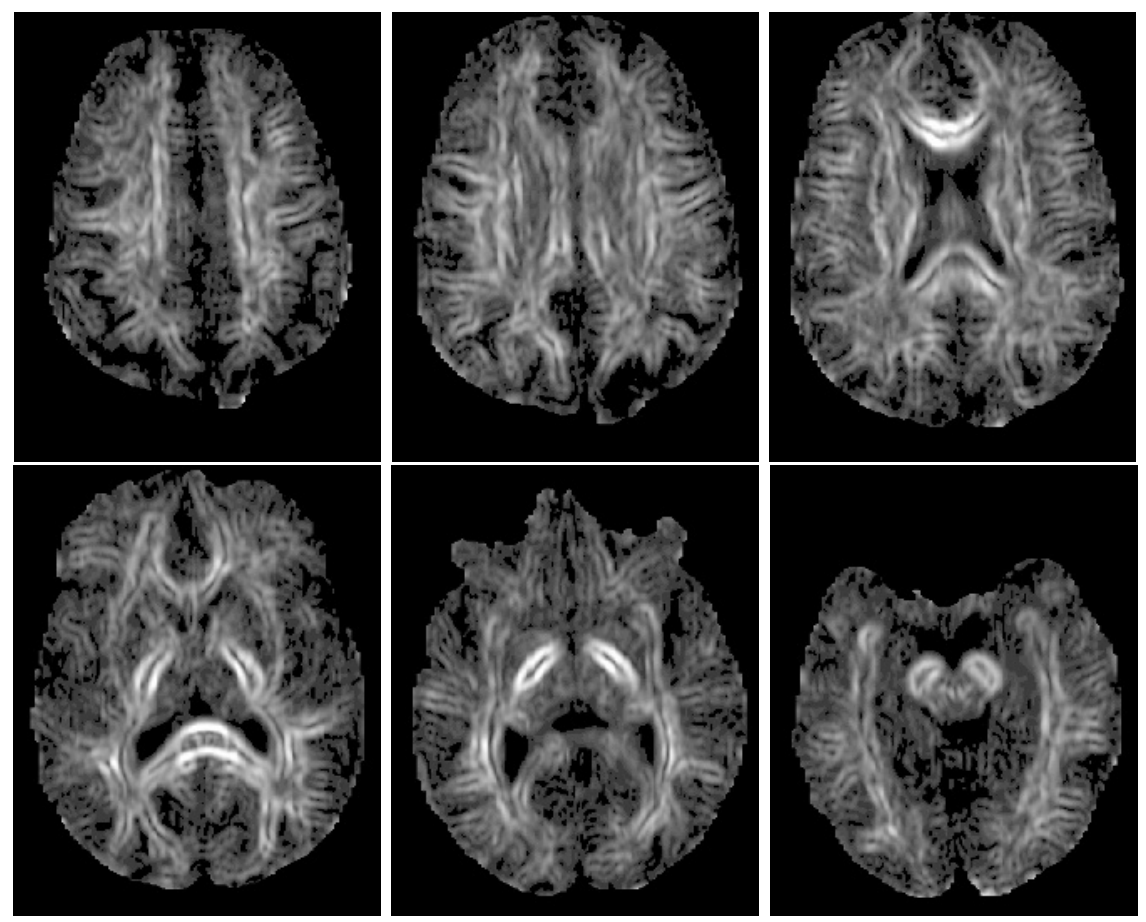

Fig. 2. Trace of the local structure tensor at several levels in an axial DTI dataset. Before filtering, the data was masked with a rough segmentation of the brain. Dark regions inside the brain, however, are not from masking but rather are regions of low edge magnitude.

One motivation for this work was interest in measuring the structure, if any, that is present in gray matter regions of a diffusion tensor dataset. The obvious choice for initial investigation is the thalamus because it is home to many nuclei which have characteristic connections to the rest of the brain, and hence some characteristic tensor orientation. We investigated the local structure in the thalamic region in three diffusion tensor datasets. The results were qualitatively similar and on some slices visually corresponded to the expected nuclear anatomy. One such slice is shown here alongside a 3D diagram of the thalamus and its nuclei.

\section{Discussion}

We have demonstrated a novel method for tissue interface detection in diffusion tensor MRI. By using a certainty field to define the importance of each tensor data point, it is possible to control the behavior of the edge detection, be insensitive to missing data, and produce subvoxel measurements. Another feature of this approach is that masks defining anatomical regions can be applied to the certainty field, removing the impact of surrounding tissue structures without 

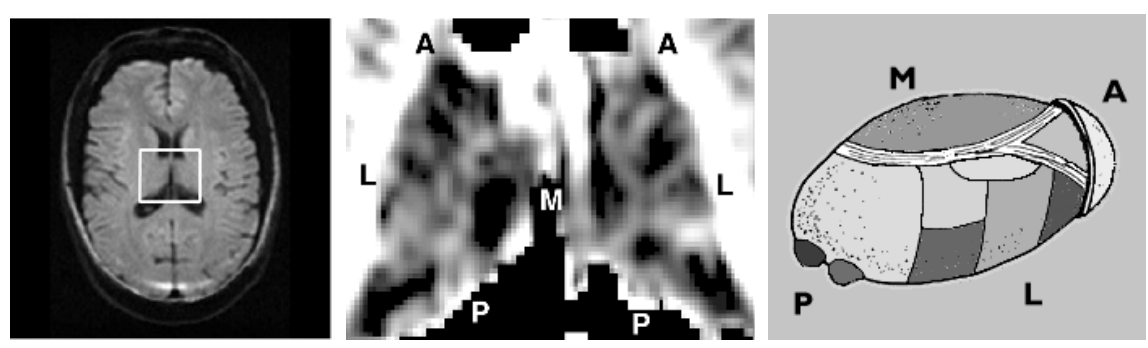

Fig. 3. DTI tissue interface detection in the region of the thalamus. The leftmost image is an axial diffusion-weighted MRI image. The white square outlines the location of the middle image, which displays the magnitude of the trace of the local structure tensor in the thalamic region. The ventricles have been masked and show in black (at the top and bottom of the image), while regions outside of the thalami with higher trace show as white. The image on the right is a diagram of the nuclei of the thalamus, adapted from www.phys.uni.torun.pl/ duch/ref/00-how-brain/. In the images the letters A, P, $\mathrm{M}$, and L signify anterior, posterior, medial, and lateral, respectively.

obtaining erroneous responses from the interface of the segmentation border. This is important since these border effects may be magnitudes stronger than the changes of interest inside the structures. Here we choose to employ the tensor trace as the certainty measure, but it would be informative to compare the behavior of the method using other measures.

The presented method is able to detect boundaries of tracts such as the optic radiation, corpus callosum, cingulate bundles, and internal capsule. In addition preliminary results demonstrate some detectable structure in the gray matter region of the thalamus, but it is not clear at this point that these interfaces represent borders between thalamic nuclei.

Acknowledgments. We would like to thank the HST Neuroimaging Training Grant, NSF ERC CISST 8810-27499, and NAC grant number NIH P41 RR 13218.

\section{References}

1. P.J. Basser, S. Pajevic, C. Pierpaoli, J. Duda, and A. Aldroubi. In vivo fiber tractography using DT-MRI data. Magnetic Resonance in Medicine, 44:625-632, 2000 .

2. TEJ Behrens, H Johansen-Berg, MW Woolrich, SM Smith, CAM WheelerKingshott, PA Boulby, GJ Barker, EL Sillery, K Sheehan, O Ciccarelli, AJ Thompson, JM Brady, and PM Matthews. Non-invasive mapping of connections between human thalamus and cortex using diffusion imaging. Nature Neuroscience, 6:750757, 2003.

3. J. Bigün, G. H. Granlund, and J. Wiklund. Multidimensional orientation: texture analysis and optical flow. IEEE Transactions on Pattern Analysis and Machine Intelligence, PAMI-13(8), August 1991. 
4. C. Feddern, J. Weickert, and B. Burgeth. Level-set methods for tensor-valued images. In O. Faugeras and N. Paragios, editors, Proc. Second IEEE Workshop on Variational, Geometric and Level Set Methods in Computer Vision, pages 65-72, 2003.

5. W. Förstner. A feature based correspondence algorithm for image matching. Int. Arch. Photogrammetry Remote Sensing, 26(3):150-166, 1986.

6. H. Gudbjartsson, S. Maier, R. Mulkern, I.A. Morocz, S. Patz, and F. Jolesz. Line scan diffusion imaging. Magnetic Resonance in Medicine, 36:509-519, 1996.

7. H. Knutsson. Representing local structure using tensors. In The 6th Scandinavian Conference on Image Analysis, pages 244-251, Oulu, Finland, June 1989.

8. H. Knutsson, H. Bårman, and L. Haglund. Robust orientation estimation in 2D, 3D and 4D using tensors. In Proceedings of Second International Conference on Automation, Robotics and Computer Vision, ICARCV'92, Singapore, September 1992.

9. H. Knutsson and C-F. Westin. Normalized and differential convolution: Methods for interpolation and filtering of incomplete and uncertain data. In Computer Vision and Pattern Recognition, pages 515- 523, 1993.

10. T.M. Koller, G. Gerig, G. Szekely, and D. Dettwiler. Multiscale detection of curvilinear structures in 2D and 3D image data. In Proc. ICCV'95, pages 864-869, 1995.

11. Rodriguez-Florido M.A., Westin C.-F., and Ruiz-Alzola J. Dt-mri regularization using anisotropic tensor field filtering. In IEEE International Symposium on Biomedical Imaging, number 336339, pages 336-339, 2004.

12. R. Deriche O. Monga, R. Lengagne. Extraction of zero crossings of the curvature derivatives in volumetric 3D medical images: a multi-scale approach. In Proc. IEEE Conf. Comp. Vision and Pattern Recognition, pages 852-855, Seattle, Washington, USA, June 1994.

13. K. Rohr. Extraction of 3D anatomical point landmarks based on invariance principles. Pattern Recognition, 32:3-15, 1999.

14. J. Ruiz-Alzola, R. Kikinis, and C-F. Westin. Detection of point landmarks in multidimensional tensor data. Signal Processing, 81:2243-2247, 2001.

15. Y. Sato, S. Nakajima, N. Shiraga, H. Atsumi, S. Yoshida, T. Koller, G. Gerig, and R. Kikinis. Three-dimensional multiscale line filter for segmentation and visualization of curvilinear structures in medical images. Medical Image Analysis, 2(2):143-168, 1998.

16. Dave S. Tuch. Diffusion MRI of Complex Tissue Structure. PhD thesis, Division of Health Sciences and Technology, Massachusetts Institute of Technology, 2002.

17. C.-F. Westin, S.E. Maier, H. Mamata, A. Nabavi, F.A. Jolesz, and R. Kikinis. Processing and visualization of diffusion tensor MRI. Medical Image Analysis, 6(2):93-108, 2002.

18. C.-F. Westin, J. Richolt, V. Moharir, and R. Kikinis. Affine adaptive filtering of CT data. Medical Image Analysis, 4(2):161-172, 2000.

19. Carl-Fredrik Westin. Multidimensional Signal Processing. PhD thesis, Linkoping University, 1994.

20. M.R. Wiegell, D.S. Tuch, H.W.B. Larson, and V.J. Wedeen. Automatic segmentation of thalamic nuclei from diffusion tensor magnetic resonance imaging. Neuroimage, 19:391-402, 2003. 